\title{
Electrical reliability and leakage mechanisms in highly resistive multiferroic $\mathrm{La}_{0.1} \mathrm{Bi}_{0.9} \mathrm{FeO}_{3}$ ceramics
}

\author{
S. Y. Wang, ${ }^{1,2, a)}$ Xue Quu, ${ }^{1}$ J. Gao, ${ }^{2}$ Yu Feng, ${ }^{1}$ W. N. Su, ${ }^{1}$ J. X. Zheng, ${ }^{1}$ D. S. Yu, ${ }^{1}$ and \\ D. J. $\mathrm{Li}^{1}$ \\ ${ }^{1}$ College of Physics and Electronic Information Science, Tianjin Normal University, Tianjin 300074, \\ People's Republic of China \\ ${ }^{2}$ Department of Physics, The University of Hong Kong, Pokfulam Road, Hong Kong
}

(Received 1 December 2010; accepted 25 March 2011; published online 13 April 2011)

\begin{abstract}
Multiferroic $\mathrm{La}_{0.1} \mathrm{Bi}_{0.9} \mathrm{FeO}_{3}$ (LBFO) ceramics with high resistivity were synthesized by using a modified rapid thermal process. The LBFO ceramics show very low leakage and low dielectric loss. Well saturated ferroelectric hysteresis loops and polarization switching currents have been observed. For a maximum applied electric field of $145 \mathrm{kV} / \mathrm{cm}$, the remanent polarization is $17.8 \mu \mathrm{C} / \mathrm{cm}^{2}$ and the coercive filed is $75 \mathrm{kV} / \mathrm{cm}$. The dominant conduction mechanism in the LBFO ceramics has been found to be the space-charge-limited current mechanism rather than the thermal excitation mechanism. Electrical reliability related to the fatigue and polarization retention of the LBFO ceramics has also been discussed with the leakage mechanisms. (c) 2011 American Institute of Physics. [doi:10.1063/1.3580604]
\end{abstract}

$\mathrm{BiFeO}_{3}$ (BFO) with perovskite structure has attracted much attention for a long time, because its high ferroelectric Curie temperature and antiferromagnetic Neel temperature is advantageous for potential application in functional devices. ${ }^{1-4}$ Theoretical studies using density function theory predict a large ferroelectric polarization of $90-100 \mu \mathrm{C} / \mathrm{cm}^{2}$ in BFO. ${ }^{5}$ However, such a large polarization was only observed in BFO thin films and single crystals. ${ }^{6,7}$ In ceramic samples, the measured polarization values are relatively low. At room temperature a remanent polarization of $11.7 \mu \mathrm{C} / \mathrm{cm}^{2}$ was obtained in a quenched $\mathrm{BiFeO}_{3}$ ceramic, ${ }^{8}$ a value of $15.5 \mu \mathrm{C} / \mathrm{cm}^{2}$ was obtained in a quenched $\mathrm{Bi}_{0.95} \mathrm{La}_{0.05} \mathrm{FeO}_{3}$ ceramic, ${ }^{9}$ and a value of $23 \mu \mathrm{C} / \mathrm{cm}^{2}$ was obtained in a high-pressure synthesized $\mathrm{BiFeO}_{3}$ ceramic. ${ }^{10}$ It has been pointed out that the difficulty in obtaining a well saturated polarization loops (P-E) and large remanent polarization comes from the large leakage current at room temperature, probably due to the presence of defects and impurities in the BFO ceramics. ${ }^{8-11}$

The requirements for the application of ferroelectric $\mathrm{BFO}$ in future devices include not only larger remanent polarization but also electrical reliability that is related to fatigue and long-term data retention. Because of pyroelectric and piezoelectric properties of the BFO ceramics, ${ }^{11,12}$ the electric cycling can induce heating effect and mechanical stresses that degrade the material, which is generally called the fatigue of the material. On the other hand, the polarization retention loss (the decrease in the remanent polarization with the elapsed time in the absence of an external electric field) is also a crucial factor that needs investigation.

The ferroelectric polarization and electrical reliability are strongly deteriorated by the leakage currents. ${ }^{4,13}$ The knowledge of the leakage current in the BFO ceramics is of fundamental interest to understand the ferroelectric performance and electrical reliability. As an added bonus, such

\footnotetext{
a) Author to whom correspondence should be addressed. Tel.: +8615822652429. FAX: +862223766519. Electronic mail: shouyu.wang@yahoo.com.
}

knowledge also allows for the characterization of the injection mechanism of charge carriers from the electrode into the materials. ${ }^{14-16}$ So far, this information has been gathered mostly in thin film and single crystal of BFO. ${ }^{2,4,14}$ Although Yuan et al. ${ }^{11}$ proposed the Poole-Frenkel conduction mechanism to be the controlling mechanism in Nd-doped BFO ceramics, Ramachandran et al. ${ }^{15}$ recently reported that the leakage current in BFO ceramics is the space-charge-limited current (SCLC). Therefore, the further study of the leakage mechanism and the influence on the electrical reliability of BFO ceramics is still necessary.

In this letter, we present the well saturated polarization loops in highly resistive $\mathrm{La}_{0.1} \mathrm{Bi}_{0.9} \mathrm{FeO}_{3}$ (LBFO) ceramics at room temperature, and furthermore the leakage mechanisms in the LBFO ceramics and their influence on the electrical reliability are investigated.

The single-phase LBFO ceramics were prepared by a modified rapid sintering process (MRSP). ${ }^{8,9}$ High purity (99.99\%) starting powders of $\mathrm{Bi}_{2} \mathrm{O}_{3}, \mathrm{Fe}_{2} \mathrm{O}_{3}$, and $\mathrm{La}_{2} \mathrm{O}_{3}$ were weighed according to the stoichiometric composition. These weighted powders were mixed in agate mortars with alcohol and milled for $5 \mathrm{~h}$. After drying, these homogeneous mixtures were calcined at $830-860{ }^{\circ} \mathrm{C}$ for $3 \mathrm{~h}$. To obtain ceramics, the calcined powder was hydrostatically cold pressed into $0.4 \mathrm{~mm}$ thick disks. Then, by using a high heating and cooling rate of about $100{ }^{\circ} \mathrm{C} / \mathrm{s}$, these pressed disks were sintered at $830-860{ }^{\circ} \mathrm{C}$ for $30 \mathrm{~min}$ in air.

The crystalline structure of the ceramics was examined by x-ray diffractometer (Rigaku D-max 2500 PC) using $\mathrm{Cu} K_{\alpha}$ radiation. For the measurement of electrical properties, both surfaces of the ceramic disks were polished and coated with silver paste as electrodes. Dielectric measurement was performed by using WK 3410 precision impedance analyzer. Leakage current, P-E loops, electrical reliability were measured with an Aixacct model TF 2000 ferroelectric analyzer.

The relative density of $\sim 90 \%$ is obtained for the sintered LBFO ceramics. The $\mathrm{x}$-ray diffraction (XRD) patterns of the ceramics were shown in Fig. 1. The XRD results indicate no 


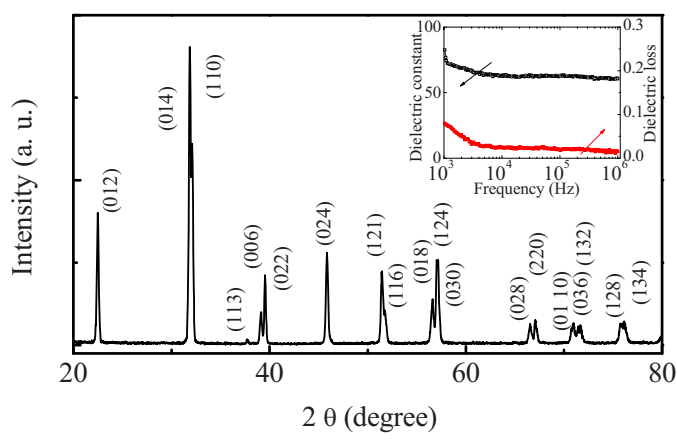

FIG. 1. (Color online) X-ray diffraction pattern of $\mathrm{La}_{0.1} \mathrm{Bi}_{0.9} \mathrm{FeO}_{3}$ ceramic. The Bragg reflections are indexed. Inset shows the dielectric constant and the dielectric loss of the ceramic as a function of the frequency.

traces of impurity phases in the LBFO ceramic samples. The XRD profile shows split reflections at $2 \theta=39.5^{\circ}$ and $57.2^{\circ}$, which indicating a rhombohedral structure in $\mathrm{La}_{0.1} \mathrm{Bi}_{0.9} \mathrm{FeO}_{3}$ ceramic. ${ }^{17}$ The variation in the dielectric constant and the dielectric loss as a function of frequency for the LBFO ceramic is shown in the inset of Fig. 1. With the increase in the frequency from $1 \mathrm{kHz}$ to $1 \mathrm{MHz}$, the dielectric constant decreases slowly from 75.1 to 53.2 and the dielectric loss decreases from $7.7 \%$ to $1.6 \%$.

Figures 2(a) and 2(b) show the displacement currents and polarization loops of the LBFO capacitor under various driving electric field, respectively. As the applied electric field is ramped to around $70-75 \mathrm{kV} \mathrm{cm}^{-1} \mathrm{~s}^{-1}$, the displacement currents exhibit a sharp increase, demonstrating the occurrence of polarization switching in the LBFO ceramics, as shown in Fig. 2(a). An applied electric field can lead to a change in the surface charges of the sample. One contribution is from the ferroelectric domain switching which can be represented by the sharp increase in the displacement currents $\mathrm{J}_{\mathrm{D}}(=\mathrm{dD} / \mathrm{dt})$. As the polarization dynamics cease after a certain time, $\mathrm{J}_{\mathrm{D}}$ subsides, and further change in the surface charge can be attributed to leakage currents through the sample, which will be discussed in the later part. If the LBFO sample is not highly resistive and the leakage currents are large to overlay the domain switching current, the sharp increases in the displacement current cannot be observed. Therefore, the observed domain switching current indicates low leakage current in the LBFO ceramics. The forward and backward coercive electric fields can be determined from the

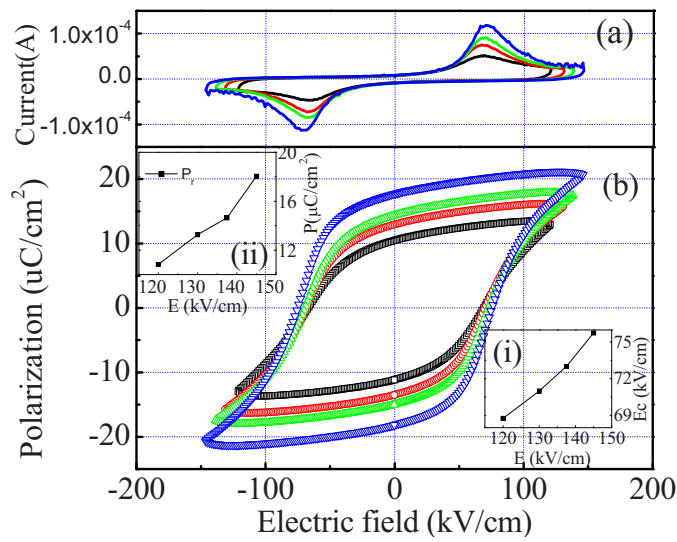

FIG. 2. (Color online) Displacement current (a) and ferroelectric polarization (b) vs electric field as measured at room temperature at a measuring frequency of $10 \mathrm{~Hz}$. Inset (i) and (ii) show the coercive field and polarization values as a function of biased electric field, respectively.
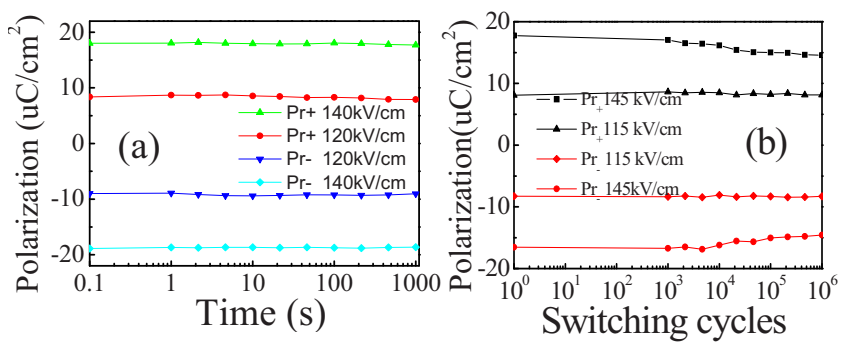

FIG. 3. (Color online) Polarization retention (a) and fatigue traits (b) of the LBFO ceramic.

current peak position (maximum current), which increases with the driving electric field, as shown in the inset (i) of Fig. 2. Moreover, the room temperature P-E response shows a well square hysteresis loops. The remanent polarization $\left(\mathrm{P}_{\mathrm{r}}\right)$ increases with the maximum driving electric field, as shown in the inset (ii) of Fig. 2. When the maximum driving filed is $145 \mathrm{kV} / \mathrm{cm}$, the measured values of the remanent polarization and the coercive field are $17.8 \mu \mathrm{C} / \mathrm{cm}^{2}$ and $75 \mathrm{kV} / \mathrm{cm}$, respectively. The remanent polarization obtained in the LBFO ceramics is significantly larger than those of some bulk ceramics and single crystal, ${ }^{8-11,18-20}$ though it is less than those of epitaxial thin films. ${ }^{1,2,4,6}$ Such a large remanent polarization in our polycrystalline ceramic is comparable to that of the $\mathrm{BiFeO}_{3}$ ceramics prepared by high-pressure synthesis. ${ }^{10}$

Figure 3(a) shows the retention of the remanent polarization of the LBFO capacitor after poling with 120 and 140 $\mathrm{kV} / \mathrm{cm}$. It can be seen that the remanent polarization does not show obvious degradation after 1000 s. Figure 3(b) shows the fatigue characteristic of the LBFO ceramic sample measured at a switching frequency of $1 \mathrm{kHz}$ and electric fields of \pm 145 and $\pm 115 \mathrm{kV} / \mathrm{cm}$. It can be seen that, after being subjected to $10^{6}$ switching cycles at $115 \mathrm{kV} / \mathrm{cm}$, there is no obvious degradation in the remanent polarization; However, for electric field of $145 \mathrm{kV} / \mathrm{cm}$, the remanent polarization shows a decrease of $17 \%$ (from 17.7 to $14.5 \mu \mathrm{C} / \mathrm{cm}^{2}$ ). It was reported that the remanent polarization decreases more than $50 \%$ only after 18 electric cycles in $\mathrm{BiFeO}_{3}$ single crystal sample, which was ascribed to the Joule heating effect induced by larger leakage current during full saturation cycles. ${ }^{7}$

Figure 4(a) shows the leakage current density versus bias electric field (J-E) up to $\pm 150 \mathrm{kV} / \mathrm{cm}$. In order to determine the leakage mechanism, J-E characteristics on a logarithmic scale and the differential curve of the $\log (\mathrm{J})$ versus $\log (\mathrm{E})$ are also plotted in Fig. 4(a). In the region of $\mathrm{E}<75 \mathrm{kV} / \mathrm{cm}$, the slope is around 1.0, thus the leakage current follows Ohmic law. In the region of $75 \mathrm{kV} / \mathrm{cm}<\mathrm{E}<150 \mathrm{kV} / \mathrm{cm}$, the slope varies from 5.0 to 2.0, indicating that the conduction mechanism may be Schottky emission, the Pool-Fenkel emission mechanism ${ }^{11,14,21}$ or the SCLC. ${ }^{15,17}$

The current density for the Schottky emission can be expressed as follows: ${ }^{21}$

$$
J_{S}=A T^{2} \exp \left[\frac{q\left(\sqrt{q V / 4 \pi \varepsilon_{0} \varepsilon_{o r} d}-\Phi\right)}{k_{B} T}\right],
$$

where $A$ is the Richardson constant, $\Phi$ is the height of the Schottky barrier, $k_{B}$ is the Boltzmann constant, $\mathrm{T}$ is the temperature in Kelvin, $\varepsilon_{o r}$ is the optical dielectric constant of the ceramic, and $d$ is the sample thickness. For the Poole- 


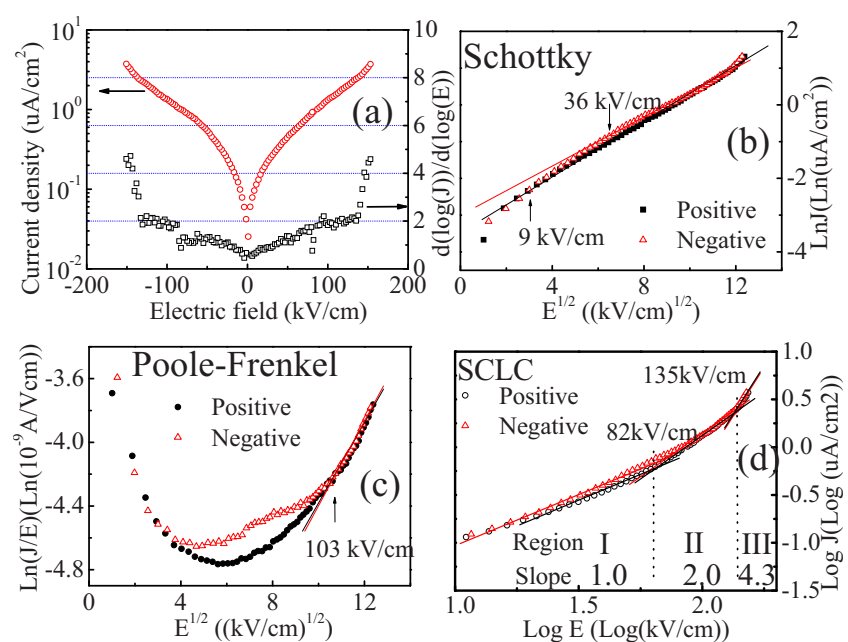

FIG. 4. (Color online) Typical J-E characteristics of the $\mathrm{La}_{0.1} \mathrm{Bi}_{0.9} \mathrm{FeO}_{3}$ ceramics at room temperature. (a) J-E characteristics on a logarithmic scale and the slope of the $\log (\mathrm{j})$ vs $\log (\mathrm{e})$ curve. Various fits of the leakage current data are shown to help determine the leakage mechanism: (b) Schottky emission, (c) Poole-Frenkel emission, or (d) SCLC.

Frenkel mechanism dominated current, $J_{P F}$ can be expressed as follows: ${ }^{21}$

$$
J_{P F}=c E \exp \left[\frac{q \sqrt{q V / \pi \varepsilon_{0} \varepsilon_{o r} d}-E_{t}}{k_{B} T}\right],
$$

where $c$ is a constant, and $E_{t}$ is the trap ionization energy.

Leakage current data for both positive and negative biases are plotted in the same scales with mentioned mechanisms, as shown Fig. 4. When the $E>9 \mathrm{kV} / \mathrm{cm}$ for positive bias or $E<-36 \mathrm{kV} / \mathrm{cm}$ for negative bias, the results can be fitted well with the Schottky equation as shown by the straight line in Fig. 4(b). When the $E>103 \mathrm{kV} / \mathrm{cm}$ for positive bias or $E<-103 \mathrm{kV} / \mathrm{cm}$ for negative bias, the PooleFrenkel mechanism also provides a good fit, as shown in Fig. 4(c). The slope of the fit lines provides an estimation of the optical dielectric constant $\varepsilon_{o r}$, and $\varepsilon_{o r}$ is thereby estimated as 0.67 and 1.56 for Schottky emission and Poole-Frenkel, respectively. These dielectric values are not in agreement with the optically determined value of about $6.25 .^{14,22}$ Therefore, at high electric field, the leakage current in the LBFO cannot be described with the Schottky emission or Poole-Frenkel leakage mechanism.

Figure 4(d) shows a plot of the $\log (\mathrm{J})$ versus $\log (\mathrm{E})$ for positive and negative bias. According to the slope, each curve can be divided into three regions. Such characteristics agree well with the SCLC theory with deep traps. ${ }^{16}$ The region I follows the Ohmic law. The region II corresponds to the trap-filling process characterized by a slope of 2.0. After the accomplishment of trap-filling, the leakage current gives a sharp increase. The conduction current in region II can be expressed as follow: ${ }^{16}$

$$
J_{\mathrm{SCLC}}=\frac{9 \mu_{p} \varepsilon_{r} \varepsilon_{0} \theta E^{2}}{8 d},
$$

where $E$ is the bias electric field, $\varepsilon_{r}$ is the permittivity of the ceramic, $\mu_{p}$ is the mobility of the charge carriers, and $\theta$ is the ratio of the total density of the induced free carriers to the trapped carriers.

For our LBFO ceramics, the MRSP may reduce the formation of charged defects, such as $\mathrm{Fe}^{2+}$ ions, $\mathrm{V}_{\mathrm{Bi}}{ }^{2+}$ vacan- cies, and $\mathrm{V}_{\mathrm{O}}{ }^{2+}$ vacancies in the samples. ${ }^{11,18-20,23,24}$ The low density of defects is a key factor to get the low leakage current and the well saturated ferroelectric hysteresis loops. However, in polycrystalline ceramic, there are still two charge-transfer bands and some traps level associating with the defects in the band gap. ${ }^{15}$ The $\mathrm{V}_{\mathrm{O}}{ }^{2+}$ vacancies act mainly as the internal traps in the polycrystalline samples, and they can move through the sample under electric fields as positive mobile charge. ${ }^{4,11}$ The charged defects, especially oxygen vacancy in LBFO ceramic could pin the ferroelectric domain wall, resulting in the decreasing of polarization during electrical switching process.

In summary, high resistive LBFO ceramics were synthesized with the MRSP method. Low leakage current, well saturated polarization loops, and excellent electrical reliability were obtained in the LBFO ceramics. The electrical conduction in the ceramics has been found to be controlled by SCLC rather than the thermal excitation mechanisms.

This work has been partly supported by the Natural Science Foundation of Tianjin (No. 11JCZDJC21800) and the Research Foundation of Tianjin Education Council.

${ }^{1}$ S.-W. Cheong and M. Mostovoy, Nature Mater. 6, 13 (2007).

${ }^{2}$ R. Ramesh and N. A. Spaldin, Nature Mater. 6, 21 (2007).

${ }^{3}$ T. Choi, S. Lee, Y. J. Choi, V. Kiryukhin, and S.-W. Cheong, Science 324, 63 (2009).

${ }^{4}$ C.-H. Yang, J. Seidel, S. Y. Kim, P. B. Rossen, P. Yu, M. Gajek, Y. H. Chu, L. W. Martin, M. B. Holcomb, Q. He, P. Maksymovych, N. Balke, S. V. Kalinin, A. P. Baddorf, S. R. Basu, M. L. Scullin, and R. Ramesh, Nature Mater. 8, 485 (2009).

${ }^{5}$ J. B. Neaton, C. Ederer, U. V. Waghmare, N. A. Spaldin, and K. M. Rabe, Phys. Rev. B 71, 014113 (2005).

${ }^{6}$ J. Wang, J. B. Neaton, H. Zheng, V. Nagarajan, S. B. Ogate, B. Liu, D. Viehland, V. Vaithyanathan, D. G. Shlom, U. V. Wahgmare, N. A. Spaldin, K. M. Rabe, M. Wuttig, and R. Ramesh, Science 299, 1719 (2003).

${ }^{7}$ D. Lebeugle, D. Colson, A. Forget, and M. Viret, Appl. Phys. Lett. 91, 022907 (2007).

${ }^{8}$ S. T. Zhang, M. H. Lu, D. Wu, Y. F. Chen, and N. B. Ming, Appl. Phys. Lett. 87, 262907 (2005).

${ }^{9}$ S. T. Zhang, Y. Zhang, M. H. Lu, C. L. Du, Y. F. Chen, Z. G. Liu, Y. Y. Zhu, N. B. Ming, and X. Q. Pan, Appl. Phys. Lett. 88, 162901 (2006).

${ }^{10}$ W. N. Su, D. H. Wang, Q. Q. Cao, Z. D. Han, J. Yin, J. R. Zhang, and Y. W. Du, Appl. Phys. Lett. 91, 092905 (2007).

${ }^{11}$ G. L. Yuan, and S. W. Or, Appl. Phys. Lett. 88, 062905 (2006).

${ }^{12}$ J. M. Liu, F. Gao, G. L. Yuan, Y. Wang, M. Zeng, and J. G. Wan, J. Electroceram. 21, 78 (2008).

${ }^{13}$ A. Morelli, S. Venkatesan, G. Palasantzas, B. J. Kooi, and J. T. M. De Hosson, J. Appl. Phys. 102, 084103 (2007).

${ }^{14}$ G. W. Pabst, L. W. Martin, Y.-H. Chu, and R. Ramesh, Appl. Phys. Lett. 90, 072902 (2007).

${ }^{15}$ B. Ramachandran, A. Dixit, R. Naik, G. Lawes, and M. S. Ramachandra Rao, Phys. Rev. B 82, 012102 (2010).

${ }^{16}$ M. A. Lampert and P. Mark, Current Injection in Solids (Academic, New York, 1970)

${ }^{17}$ A. V. Zalesskii, A. A. Frolov, T. A. Khimich, and A. A. Bush, Phys. Solid State 45, 141 (2003).

${ }^{18}$ Y. P. Wang, L. Zhou, M. F. Zhang, X. Y. Chen, J.-M. Liu, and Z. G. Liu, Appl. Phys. Lett. 84, 1731 (2004).

${ }^{19}$ B. F. Yu, M. Y. Li, J. Wang, L. Pei, D. Y. Guo, and X. Z. Zhao, J. Phys. D 41, 185401 (2008).

${ }^{20}$ Y. Wang and C. W. Nan, Appl. Phys. Lett. 89, 052903 (2006).

${ }^{21}$ S. M. Sze, Physics of Semiconductor Devices, 2nd ed. (Wiley, New York, 1981).

${ }^{22}$ S. Iakovlev, C.-H. Solterbeck, M. Kuhnke, and M. Es-Souni, J. Appl. Phys. 97, 094901 (2005).

${ }^{23}$ M. Kumar and K. L. Yadav, Appl. Phys. Lett. 91, 242901 (2007).

${ }^{24}$ D. K. Pradhan, R. N. P. Choudhary, C. Rinaldi, and R. S. Katiyar, J. Appl. Phys. 106, 024102 (2009). 